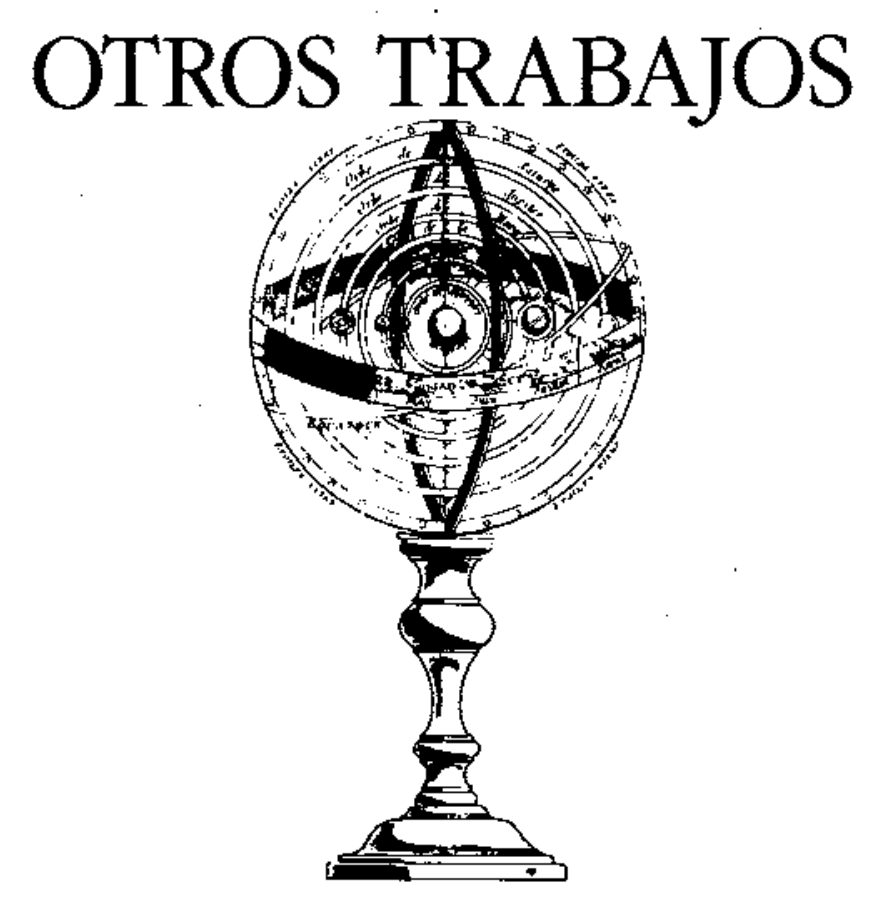

\title{
¿SABEN LOS MAESTROS LA FÍSICA QUE ENSENAN?
}

\author{
ZALAMEA GODOY, E. y PARÍS ESPINOSA, R. \\ Universidad Nacional de Colombia. Universidad Distrital F.J. de Caldas.
}

\section{SUMMARY}

This paper attempts to report a research carried out in Colombia about the proficiency in mechanics of high school teachers. Based on observations, some judgements and appreciations are made about the teachers' performance and a teaching method proposal is suggested in order to solve the problems which have been identified.

\section{INTRODUCCIÓN}

Este trabajo informa sobre una investigación realizada en una amplia población de profesores de física de nivel medio en varios cursos de capacitación. Estas circunstancias nos permitieron indagar acerca de la solidez en la conceptualización y manejo por parte de los maestros de los elementos que consideramos esenciales en la teoría de la mecánica newtoniana. La forma de trabajo fue la siguiente: En cada cursillo se planteaba a los maestros una circunstancia real y se les formulaba una pregunta aparentamente simple acerca de esa situación que llamaremos "problema-experimento", y que se describirá posteriormente. En grupos pequeños los profesores discutían sus soluciones. A continuación cada grupo exponía sus conclusiones, originándose un debate alrededor del problema. Como en todos los debates, sucedió que las soluciones de los diferentes grupos fueron ampliamente contradictorias; se generaba una intensa discusión, sin llegar en ningún caso a una solución unánime. 
Agotadas las posibilidades de diálogo se les planteaba un segundo problema-experimento, también eminentemente real.

Se suscitaba una nueva discusión y al final solo persistían dos soluciones una de ellas marcadamente mayoritaria. Entonces se procedía a llevar a cabo la experiencia con un resultado sorpresivo pues los maestros se deban cuenta que sus predicciones estaban erradas con lo cual la discusión cobraba nueva intensidad. Aunque en muchas ocasiones se acercaron a la explicación correcta, nunca llegaron a ella. Es de enfatizar que los 273 profesores entrevistados en cinco capitales de provincias son personas preocupadas tanto por el mejoramiento de los contenidos teóricos como por la calidad de su docencia.

\section{LOS DOS PROBLEMAS-EXPERIMENTO}

El contenido de la primera situación es el siguiente: Se tiene un bloque de madera sobre una mesa plana y horizontal. Si le aplicamos una fuerza $\mathbf{F}$, horizontal y no se desplaza es porque el piso le aplica una fuerza de rozamiento estático $f_{s}$. Se ha de construir una gráfica de $f_{s}$ contra $F_{a}$, desde $F_{a}$ vale cero hasta cuando el bloque comienza a desplazarse. Queremos indicar la solución de la cuestión ya que esto nos permitirá mayor comprensión de los resultados obtenidos y de nuestros posteriores análisis. La gráfica es una recta a $45^{\circ}$; puesto que el bloque no cambia su estado de movimiento, la fuerza neta sobre él debe ser igual a $\mathrm{O}$. Recordemos además que es imposible medir la fuerza $f_{s}$; solo la teoría newtoniana nos permite deducir su valor.

En el segundo problema-experimento planteábamos Io siguiente: Sobre una mesa colocamos varias hojas de papel periódico y sobre éstas un ladrillo, un borrador de tablero y una moneda, como lo esquematiza la figura 1. A continuación se anunciaba que alguien iba a coger las hojas de periódico por su extremo colgante y que les aplicaría un violento tirón horizontal. Se solicitaba que predijesen cómo irían a quedar colocados sobre la mesa los tres cuerpos. Nos limitamos ahora a contar lo que se observa al llevar a cabo la experiencia descrita. El

figura 1

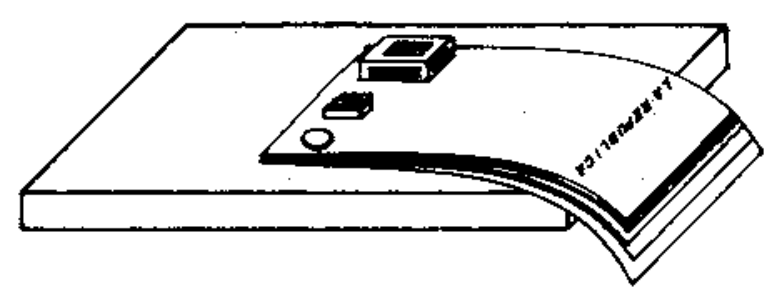

cuerpo que más se desplaza es el ladrillo, luego sigue el borrador de tablero y el que menos se desplaza es la moneda.

\section{3. ¿POR QUÉ ESTAS DOS SITUACIONES?}

Nuestra experiencia de maestros de mecánica en los primeros semestres de Universidad nos ha permitido detectar un conjunto de malformaciones de la teoría relativa al movimiento en los estudiantes que inician su formación profesional (Zalamea 1983, París 1986). Más aún, exámenes detallados han conducido a afirmar que las explicaciones de un alumno acerca del movimiento antes de recibir la física del Bachillerato persisten después de los años finales de la escuela secundaria (Gil 1983, Gilbert y Watts 1983). Por ello en más de una ocasión nos preguntábamos si se podría garantizar que los maestros de Bachillerato hayan procesado los postulados newtonianos. Con esta duda en mente diseñamos los problema-experimento que acabamos de describir. Considerados de una manera general, nos permitirian constatar la firmeza y consistencia de la asimilación de la mecánica por parte de los maestros, y detectar si existen o no fallas comunes en sus formas de interpretación, explicación y aplicación de esas leyes.

Nuestras intenciones iban más allá. Estamos convencidos de que una de las causas por las cuales una persona, en general, no procesa una información relativa a las leyes del movimiento, es por la ausencia de oportunidades concretas para confrontar los contenidos de esa información con sus formas innatas de interpretación (Gil 1983). Consideramos que esta confrontación es ineludible en el proceso de sustitución del conocimiento común por el conocimiento científico, pues es la reflexión sobre los resultados de esa confrontación la que permite invalidar las explicaciones innatas y aquilatar y dar sentido a las teorías científicas. Con base en esta idea compartimos la nueva visión de la didáctica de la física, en donde el papel del maestro como poseedor y distribuidor de la verdad se relega a un plano muy secundario (Gil 1983), mientras se destaca su oficio de guía de los procesos de aprendizaje de los alumnos a través de problemas-experimento cuidadosamente escogidos, estudiados y analizados. No queremos pecar de illusos permitiendo dar a entender que un alumno llega por sí solo a formalizar alguna Teoría Física como tal; estamos convencidos de que ello es casi imposible (Gil 1983). Pero una vez que en la práctica un estudiante ha podido constatar la inconsistencia de sus preconcepciones, está ante la posibilidad de empezar a procesar de manera crítica y fecunda la información del maestro, la cual no debe iniciar el proceso sino concluirlo.

\section{RESULTADOS DE LA PRIMERA SITUACIÓN}

Queremos recalcar que no vale la pena discriminar las opiniones por cursillos, ni realizar sobre ellas estadísti- 
cas, pues uno de sus elementos esenciales es la uniformidad en todos los eventos. Así pues, invariablemente en todas partes los participantes construyeron los siguientes gráficos:

figura 2
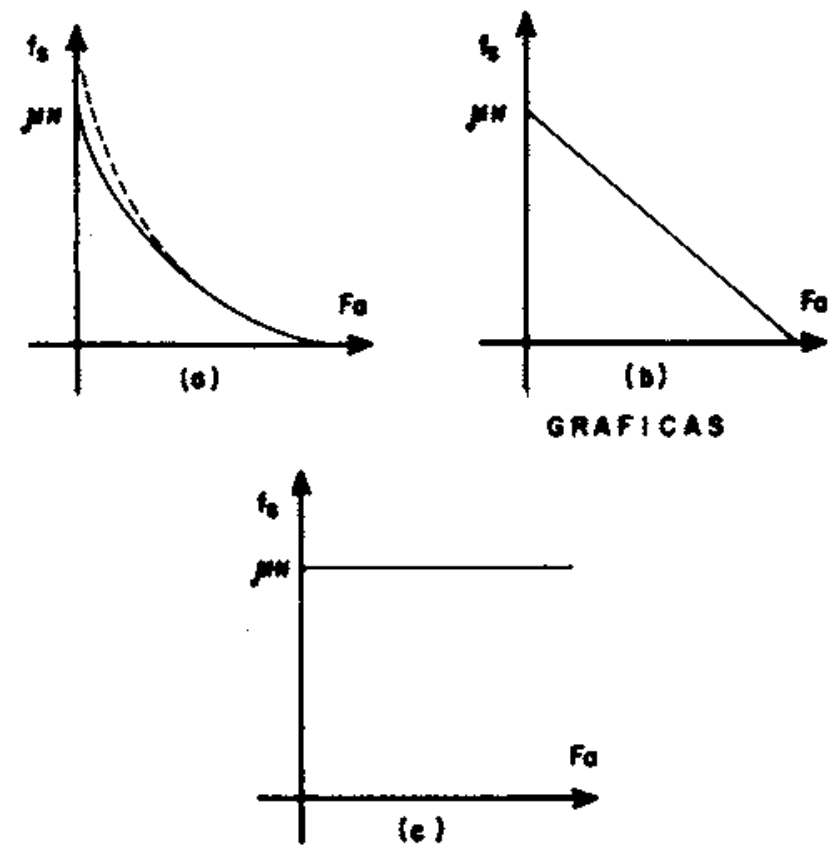

Los argumentos mediante los cuales cada grupo sustentaba sus opiniones también fueron idénticos. Los defensores de las gráficas (a) y (b) sostenían que tan pronto se aplica la fuerza $F_{z}$ aparece una fuerza de rozamiento máximo $f_{s}$ para que el cuerpo no se desplace. A medida que se aumenta el valor de la fuerza $F_{\mathrm{a}}$ la fuerza de rozamiento va disminuyendo, y es en la forma del decrecimiento como se diferencian; para los unos es exponencial y para otros lineal. Finalmente los dos grupos concliyen que cuando la fuerza de rozamiento $f_{s}$ es cero comienza a deslizarse. El grupo minoritario que defen * día la variante de la gráfica (a) sostenía que antes de aplicar la fuerza $F_{a}$ existía la fricción actuando sobre el bloque y que su valor era inmenso. Los grupos que planteaban como solución a lo propuesto la gráfica (c) sostenían que en todo momento desde que comienza a aplicarse la fuerza $F_{a}$, la de rozamiento es constante, y que cuando $F_{\mathbf{z}}$ es mayor el bloque se desliza. Afirmaban que la fuerza de rozamiento no variaba, apoyados en la relación $f_{s}=\mu_{s} N$. Como $N$ no varía y $\mu_{s}$ no varía, $f_{s}$ no puede variar. Nuestra actuación durante estos debates se limitaba a mantener la discusion recalcando los puntos contradictorios de las diferentes posiciones. Es evidente que las concepciones que sustentaban estas gráficas están en abierta contradicción con las leyes de la dinámica que enseñan los maestros.

Las principales alternativas mediante las cuales los maestros se defendían de nuestras objecciones son: E1 bloque no se mueve a pesar de haber fuerza neta sobre él porque las fuerzas de cohesion lo sostienen aferrado al piso; a esta explicación la llamamos en los diferentes eventos la "teoría del pegante". Otra frecuente alternativa era: El cuerpo no se mueve porque no se Ie aplica la fuerza necesaria para moverlo. Esta opinión se entremezclaba con otra, que expresamos no con las palabras de los maestros, que siempre nos parecieron muy confusas, sino respetando la esencia de la idea que ellos emitían: "La suma $F_{2}+f_{\text {s }}$ no es cero, lo que es cero es esa suma, más Ia fuerza que yo le aplico (es decir $\mathbf{F}_{\mathbf{g}}$ )". Por otra parte los defensores de la gráfica (c), continuaban aferrados a sus argumentos algebraicos, a pesar de que algunos colegas les hacían notar que la formulación correcta de la ecuación en que se apoyaban era $f_{s}$ $\leq \mu_{s} N$. Nunca faltaron los que opinaban que inicialmente la fuerza actuaba verticalmente, con lo cual se explicaban que el bloque se "aferraba" al piso. En dos ocasiones algunos maestros llegaron a concluir que en situaciones estáticas no son aplicables las leyes de Newton, convenciendo de ello a muchos de sus colegas. Frecuentemente, una vez iniciada la discusión por grupos, algunos preguntaban por dinamómetros y bloques para poder realizar las gráficas; inmediatamente se les entregaban y notábamos con atención las caras de asombro ante la imposibilidad de "medir" lo que pretendian.

\section{RESULTADOS DE LA SEGUNDA SITUACION}

Una vez anunciado ei segundo problema-experimento algunos participantes exclamaban: "Eso no se puede resolver, faltan datos". "¿Qué datos les faltan?", preguntábamos. Contestaban: "¿Cómo es la superficie? ¿Cómo es el papel? ¿Cuál ladrillo?” Les entregábamos entonces el ladrillo, el borrador, la moneda y el periódico, pero les pedíamos que no realizaran la experiencia antes de enunciar sus predicciones. Iniciada la discusión por grupos el entusiasmo de los maestros era evidente; sentían confianza en sí mismos; al cabo de cinco minutos ya no había discusión. La mayoría opinaba: "Si el tirón es violento ninguno de los tres cuerpos se mueve, porque los cuerpos tienden a permanecer en reposo; esos cuerpos están en reposo y mientras nadie los empuje tienden a conservarlo por la inercia de los cuerpos". La seguridad y confianza de los maestros es una característica digna de anotar. Por otra parte un grupo minoritario $(35 \%)$ sostenía que era posible que los cuerpos se movieran. Había entre ellos quienes opinaban que los tres cuerpos se moverían muy poco pero todos igual; los restantes opinaban que el ladrillo casi no se movería, que el borrador avanzaría un poquito, pero que la moneda avanzaría notoriamente. ¿Por qué? Les preguntábamos a los unos y a los otros. "Por inercia", respondían a coro, y los últimos añadían: "El ladrillo tiene más inercia porque tiene más masa y entonces tiende más que el borrador o la moneda a permanecer en el estado de reposo en que está. La moneda tiene muy poca inercia y entonces es la que más se mueve". Ante estos hechos presentábamos la siguiente alternativa de discusión: hay dos opiniones que evidentemente no se concilian; ¿en qué está equivoca- 
đo el bando oponente? Rápidamente se agotaba la discusion, los diferentes grupos mantenían sus opiniones. Procedíamos entonces a realizar la experiencia. Pasada la sorpresa, reiniciábamos la discusión con las preguntas: ¿Cómo nos explicamos lo que acabamos de observar? ¿Por qué nos equivocamos en nuestra predicción? Muchos de los participantes en los diferentes eventos comenzaban a pensar correctamente que la fricción det papel sobre cada cuerpo era un elemento que habían olvidado en sus análisis. Sin embargo, la acentuada idea de "inercia" les impedía aproximarse a análisis más correctos. Se presentaron además dos obstáculos para el éxito de esta aproximación, a saber: Acabamos de decir unas líneas más arriba "la fricción del papel sobre cada cuerpo", pero no es ésta la forma de expresión de los maestros. Ellos dicen: "La fuerza de fricción entre el papel y los cuerpos", con lo cual parece que no identifican claramente quién aplica la fuerza sobre quién (tercera ley) y es notoria la ambigüedad y confusión entre la cualidad "ser rugoso" y la cantidad física "fuerza de rozamiento", de tal forma que para muchos estas dos cosas "generan" dos fuerzas distintas. EI segundo obstáculo se basa en la consideración, altamente frecuente, de que la fuerza que se le aplica al periódico se "transmite íntegramente" a cada uno de Ios tres cuerpos. Cuando las discusiones estaban a punto de agotarse y máxime si había quiénes nos parecía que estaban próximos a una solución correcta, planteábamos una variante diciéndoles: "Si ahora tomamos el ladrillo que hemos utilizado y medio ladrillo del mismo material, los alineamos sobre el periódico y jalamos violentamente, ¿cuál se desplazaría más?" Invariablemente la respuesta era "el de mayor masa se mueve menos porque tiene más inercia". AI realizar la experiencia los dos ladrillos avanzan prácticamente lo mismo; esto constituye un nuevo conflicto generador para los maestros, en el sentido de que los conduce a replantearse la validez de sus afirmaciones y a reexaminar críticamente el conjunto de "definiciones y leyes" que ellos manejan como verdades absolutas. Así pues, cuando hacíamos notar a los maestros que si ellos definen masa como la medida de la inercia y que si utilizan en sus clases estas experiencias para comprobar que los cuerpos tienden a oponerse a cambiar su estado de reposo, deberían concluir que la moneda posee mayor inercia y por lo tanto mayor masa, puesto que avanza menos y presenta mayor "oposición" a cambiar su estado de reposo. Con cierto grado de desesperación decían: Entonces, ¿está mal definida la masa? ¿Qué es inercia y qué nos queda de la primera ley đe Newton? Aquí suspendíamos los debates. Al día siguiente o por lo menos después de un buen descanso, tomábamos la palabra y exponíamos nuestra concepción acerca de las leyes de Newton, y la aplicación de esas leyes a las dos situaciones.

\section{ANÁLISIS Y CONCLUSIONES}

- Aun cuando el propósito inicial de los diferentes eventos era la realización de cursos de capacitación para profesores de física en secundaria, los hechos observados que hemos descrito nos dan pie para emitir algunos juicios. La primera inquietud que asalta a quien ha leído este informe debe estar relacionada con la pregunta: "¿Saben los maestros la física que enseñan? La respuesta a este comprometedor interrogante implica una investigación de mayor envergadura que la presente, así los resultados obtenidos nos dejan una cierta duda al respecto. Pero hay algunos hechos que no queremos dejar pasar desapercibidos, ya que contribuyen por lo menos a aumentar la duda. No nos parece grave que un maestro aisladamente analice en forma equivocada los dos problemas-experimento, pero nos alarma que el análisis en grupos también sea erróneo, pues en ellos cada maestro repite ante sus colegas los procesos que posiblemente realiza en sus clases para "enseñar" a sus alumnos. Junto a esto anotamos que los maestros no se dan cuenta, ni individual ni colectivamente, de las incongruencias entre sus opiniones y las teorías de la mecánica newtoniana, y que no valoran la importancia y trascendencia de estas incongruencias. Todo esto conduce a dudar de la solidez del conocimiento que puedan tener de la mecánica clásica. La situación parece agravarse si la miramos desde otro punto de vista. Los maestros están familiarizados con la información relativa a la mecánica clásica; pueden repetir, la mayoría de las veces correctamente, los postulados, las leyes fundamentales y las ecuaciones algebraicas en ellas involucradas; pero ese cuerpo teórico no les sirve para interpretar una situación concreta. Parece que en ellos predomina un aprendizaje memorístico, puntual e inconexo, en el sentido siguiente: tal ley dice tal cosa y se cumple en tal caso; tal otra ley dice tal otra cosa y se cumple en tal otro caso, y tal ecuación dice..., etc. Es por esto que para ellos no hay problema en que se infrinjan las leyes de Newton con tal de salvar la ecuación de la fuerza de rozamiento estático. Tal vez es también por esto que ante una situación nueva (o presentada de una forna nueva), las primeras palabras que pronuncian son: "Ah!, eso es por inercia"; como buscando encasillar el fenómeno en el cajón que le corresponde para servir de ejemplo y no, como nos parece que debe ser, buscando que todo el conjunto teórico les proporcione una explicación de la situación. Es de suponer que esta forma de concebir la física influencie los procesos de enseñanza-aprendizaje que ellos orientan.

- Un aspecto complementario es la marcada inclinación manifestada por los maestros a considerar las fórmulas como parte esencial de la teoría; constatamos que para muchos es más importante la fórmula de la fuerza de rozamiento estático que la segunda ley de Newton, y no encuentran dificultad en negar el cumplimiento de ésta con tal de salvar aquélla.

- Conviene expresar una diferenciación en el diseño de los dos problemas-experimento. El primero lo concebimos con la pretensión de que fuera novedoso para el maestro y por consiguiente una apropiada ocasión para poner a prueba la solidez de sus construcciones teóricas, pues consideramos que alguien sabe una teoría si la aplica correctamente en una situación no estudiada antes. Por el contrario sabíamos que el segundo problema-experimento era familiar al maestro, pues no solo aparece en textos escolares sino incluso en libros de 
divulgación científica popular como "La Física Recreativa" (Perelman 1962) para ilustrar seudoparadigmas que acríticamente se han transmitido de generación en generación. Así pues la realización de la experiencia con resultados contrarios a los enseñados tradicionalmente por textos y maestros, se convierte en un conflicto (Bachelard 1979) que conduce al docente a una revisión y reelaboración de las informaciones recibidas pasivamente; era de esperar, que nuevamente la teoría como un todo iluminara su análisis, lo cual como hemos anotado no sucedió.

- Las actitudes adoptadas por los maestros pretendían medir algo o realizar un experimento, nos dan ocasión para emitir algunos juicios al respecto. Ante la imposibilidad de solucionar la primera situación el maestro acude al experimento y a la medición con una salvadora esperanza: los datos le darán la respuesta. Inconscientemente rememora la equivocada concepción de las tradicionales "guías de laboratorio" que Ie condujeron a una idea falseada y simplista del quehacer del físico: para hacer física basta tomar datos, hacer gráficas, y de ellas saldrán infaliblemente las leyes físicas. En esta concepción la medida es un medio para llegar a la teoría. Como no estamos de acuerdo con esto diseñamos el primer problema-experimento con Ia idea de hacerle ver al maestro que es la teoría, la física, la que da sentido a una medición o a un experimento; y que el mecanismo de la medición también está orientado por la teoría.

- Analicemos otro aspecto. Varias investigaciones recientes (Gilbert Watts 1983, Viennot 1979, Clement 1982), han establecido que antes de la acción de la escuela los estudiantes poseen algunas concepciones explicativas las cuales son diferentes de las teorias científicas y se constituyen en un obstáculo epistemológico (Bachelard 1979) para que los alumnos procesen y asimilen teorías válidas. Pues bien, hemos hallado que algunos de estos elementos preteóricos aún persisten en la mayoría de los maestros y que se complementan negativamente con un conjunto de "paradigmas" que son malformaciones de la teoría correcta. Manifiestan concepciones preteóricas cuando afirman que las fuerzas se "transmiten" de un cuerpo a otro, o que existen "fuerzas

\section{REFERENCIAS BIBLIOGRÁFICAS}

BACHELARD, G., 1979. La formación del espíritu científico. (Siglo XXI: Buenos Aires).

CLEMENT, J., 1982. Student's preconceptions introductory mechanics, American Journal of Physics. Vol. 50(I) pp. 66-71. mínimas" dependientes del cuerpo y no de su entorno para sacarlo del reposo, o que la fuerza neta actúa en la dirección de la velocidad; y malformaciones de la teórica cuando afirman que las fuerzas de fricción "siempre" se oponen al movimiento de los cuerpos, o que debe existir "fuerza aplicada" en la dirección de movimiento de un cuerpo o que "la masa es la propiedad que tienen los cuerpos para no dejarse variar su estado de movimiento".

- Hemos afirmado que la causa por la cual una persona no procesa y asimila un determinado aspecto del saber, referente a las ciencias naturales, es la falta de oportunidades para confrontar las informaciones teóricas con el acaecer cotidiano, y por la falta de reflexión crítica sobre esas informaciones. Es decir, en la medida que el estudiante (futuro maestro) reciba los contenidos como verdades ya establecidas y de una manera pasiva en cuanto a cuestionarlas se refiere, aunque muy activa en cuanto a aplicarlos en situaciones estereotipadas y generalmente alejados de la realidad (problemas "tipo texto"), en esa medida su asimilación será superficial y ante una situación nueva y problemática no puede acudir a la teoría como un conjunto, sino a la aplicación inconexa de "verdades" que le funcionaron en los ejemplos o situaciones estereotipadas. Con base a estas consideraciones estructuramos los diferentes encuentros con maestros de física que hemos comentado. Proponemos esta forma de trabajo para cursos de capacitación de profesores de la escuela secundaria a fin de someterlo a críticas y evaluaciones más profundas de las que nosotros hemos realizado.

Pretendemos colocar colectivamente a los maestros ante situaciones reales en donde hay una pregunta o una cuestión que conlleve la aplicación de una teoría completa de alguna parte de la física, a fin de que en el proceso de solución se vean obligados a cuestionar las afirmaciones de los demás y a evaluar críticamente los rangos de validez de sus afirmaciones. Creemos que $\mathrm{el}$ método es conveniente para la enseñanza de la física en los primeros semestres de la Universidad y más aún para estudiantes de nivel medio (Bachillerato). 
PARÍS, R, 1986. Análisis de situaciones dinámicas en las que fuerzas aplicadas no producen movimiento. U.P.N. Tesis.

PERELMAN, Y., 1962. Física recreativa. (Mir: Moscú).

PIAGET, J., 1969. Psicología y Pedagogía. (Ariel: Barcelona).
VIENNOT, L., 1979. Spontaneous reasoning in elementary dinamics, European Journal of Science Education, Vol. 1, pp. 205-221.

ZALAMEA, E., 1983. Dificultades en el aprendizaje de la primera ley de Newton U.P.N. Tesis. 